\title{
Existence of Pseudo Almost Automorphic Solutions for the Heat Equation with $S^{p}$-Pseudo Almost Automorphic Coefficients
}

\author{
Toka Diagana ${ }^{1}$ and Ravi P. Agarwal ${ }^{2}$ \\ ${ }^{1}$ Department of Mathematics, Howard University, 2441 6th Street NW, Washington, DC 20005, USA \\ ${ }^{2}$ Department of Mathematical Sciences, Florida Institute of Technology, Melbourne, FL 32901, USA
}

Correspondence should be addressed to Ravi P. Agarwal, agarwal@fit.edu

Received 12 March 2009; Accepted 3 July 2009

Recommended by Veli Shakhmurov

We obtain the existence of pseudo almost automorphic solutions to the $N$-dimensional heat equation with $S^{p}$-pseudo almost automorphic coefficients.

Copyright (C) 2009 T. Diagana and R. P. Agarwal. This is an open access article distributed under the Creative Commons Attribution License, which permits unrestricted use, distribution, and reproduction in any medium, provided the original work is properly cited.

\section{Introduction}

Let $\Omega \subset \mathbf{R}^{N}(N \geq 1)$ be an open bounded subset with $C^{2}$ boundary $\partial \Omega$, and let $\mathbf{X}=\mathbf{L}^{2}(\Omega)$ be the space square integrable functions equipped with its natural $\|\cdot\|_{\mathbf{L}^{2}(\Omega)}$ topology. Of concern is the study of pseudo almost automorphic solutions to the $N$-dimensional heat equation with divergence terms

$$
\begin{aligned}
\frac{\partial}{\partial t}[\varphi+F(t, \widehat{B} \varphi)] & =\Delta \varphi+G(t, \widehat{B} \varphi), \quad t \in \mathbf{R}, x \in \Omega \\
\varphi(t, x) & =0, \quad t \in \mathbf{R}, x \in \partial \Omega,
\end{aligned}
$$

where the symbols $\widehat{B}$ and $\Delta$ stand, respectively, for the first- and second-order differential operators defined by

$$
\widehat{B}:=\sum_{j=1}^{N} \frac{\partial}{\partial x_{j}}, \quad \Delta=\sum_{j=1}^{N} \frac{\partial^{2}}{\partial x_{j}^{2}}
$$

and the coefficients $F, G: \mathbf{R} \times \mathbf{H}_{0}^{1}(\Omega) \mapsto \mathbf{L}^{2}(\Omega)$ are $S^{p}$-pseudo almost automorphic. 
To analyze (1.1), our strategy will consist of studying the existence of pseudo almost automorphic solutions to the class of partial hyperbolic differential equations

$$
\frac{d}{d t}[u(t)+f(t, B u(t))]=A u(t)+g(t, C u(t)), \quad t \in \mathbf{R},
$$

where $A: D(A) \subset \mathbf{X} \mapsto \mathbf{X}$ is a sectorial linear operator on a Banach space $\mathbf{X}$ whose corresponding analytic semigroup $(T(t))_{t \geq 0}$ is hyperbolic; that is, $\sigma(A) \cap i \mathbf{R}=\emptyset$, the operator $B, C$ are arbitrary linear (possibly unbounded) operators on $\mathbf{X}$, and $f, g$ are $S^{p}$-pseudo almost automorphic for $p>1$ and jointly continuous functions.

Indeed, letting $A \varphi=\Delta \varphi$ for all $\varphi \in D(A)=\mathbf{H}_{0}^{1}(\Omega) \cap \mathbf{H}^{2}(\Omega), \widehat{B} \varphi=B \varphi=C \varphi$ for all $\varphi \in \mathbf{H}_{0}^{1}(\Omega)$, and $f=F$ and $g=G$, one can readily see that (1.1) is a particular case of (1.3).

The concept of pseudo almost automorphy, which is the central tool here, was recently introduced in literature by Liang et al. [1,2]. The pseudo almost automorphy is a generalization of both the classical almost automorphy due to Bochner [3] and that of pseudo almost periodicity due to Zhang [4-6]. It has recently generated several developments and extensions. For the most recent developments, we refer the reader to [1, 2, 7-9]. More recently, in Diagana [7], the concept of $S^{p}$-pseudo almost automorphy (or Stepanov-like pseudo almost automorphy) was introduced. It should be mentioned that the $S^{p}$-pseudo almost automorphy is a natural generalization of the notion of pseudo almost automorphy.

In this paper, we will make extensive use of the concept of $S^{p}$-pseudo almost automorphy combined with the techniques of hyperbolic semigroups to study the existence of pseudo almost automorphic solutions to the class of partial hyperbolic differential equations appearing in (1.3) and then to the $N$-dimensional heat equation (1.1).

In this paper, as in the recent papers [10-12], we consider a general intermediate space $\mathbf{X}_{\alpha}$ between $D(A)$ and $\mathbf{X}$. In contrast with the fractional power spaces considered in some recent papers by Diagana [13], the interpolation and Hölder spaces, for instance, depend only on $D(A)$ and $\mathbf{X}$ and can be explicitly expressed in many concrete cases. Literature related to those intermediate spaces is very extensive; in particular, we refer the reader to the excellent book by Lunardi [14], which contains a comprehensive presentation on this topic and related issues.

Existence results related to pseudo almost periodic and almost automorphic solutions to the partial hyperbolic differential equations of the form (1.3) have recently been established in $[12,15-18]$, respectively. Though to the best of our knowledge, the existence of pseudo almost automorphic solutions to the heat equation (1.1) in the case when the coefficients $f, g$ are $S^{p}$-pseudo almost automorphic is an untreated original problem and constitutes the main motivation of the present paper.

\section{Preliminaries}

Let $(\mathbf{X},\|\cdot\|),\left(\mathbf{Y},\|\cdot\|_{\mathbf{Y}}\right)$ be two Banach spaces. Let $B C(\mathbf{R}, \mathbf{X})($ resp., $B C(\mathbf{R} \times \mathbf{Y}, \mathbf{X}))$ denote the collection of all $\mathrm{X}$-valued bounded continuous functions (resp., the class of jointly bounded continuous functions $F: \mathbf{R} \times \mathbf{Y} \mapsto \mathbf{X})$. The space $B C(\mathbf{R}, \mathbf{X})$ equipped with the sup norm $\|\cdot\|_{\infty}$ is a Banach space. Furthermore, $C(\mathbf{R}, \mathbf{Y})$ (resp., $C(\mathbf{R} \times \mathbf{Y}, \mathbf{X})$ ) denotes the class of continuous functions from $\mathbf{R}$ into $\mathbf{Y}$ (resp., the class of jointly continuous functions $F: \mathbf{R} \times \mathbf{Y} \mapsto \mathbf{X}$ ). 
The notation $L(\mathbf{X}, \mathbf{Y})$ stands for the Banach space of bounded linear operators from $\mathbf{X}$ into $Y$ equipped with its natural topology; in particular, this is simply denoted $L(\mathbf{X})$ whenever $\mathrm{X}=\mathrm{Y}$.

Definition 2.1 (see [19]). The Bochner transform $f^{b}(t, s), t \in \mathbf{R}, s \in[0,1]$ of a function $f: \mathbf{R} \mapsto$ $\mathbf{X}$ is defined by $f^{b}(t, s):=f(t+s)$.

Remark 2.2. (i) A function $\varphi(t, s), t \in \mathbf{R}, s \in[0,1]$, is the Bochner transform of a certain function $f, \varphi(t, s)=f^{b}(t, s)$, if and only if $\varphi(t+\tau, s-\tau)=\varphi(s, t)$ for all $t \in \mathbf{R}, s \in[0,1]$ and $\tau \in[s-1, s]$.

(ii) Note that if $f=h+\varphi$, then $f^{b}=h^{b}+\varphi^{b}$. Moreover, $(\lambda f)^{b}=\lambda f^{b}$ for each scalar $\lambda$.

Definition 2.3. The Bochner transform $F^{b}(t, s, u), t \in \mathbf{R}, s \in[0,1], u \in \mathbf{X}$ of a function $F(t, u)$ on $\mathbf{R} \times \mathbf{X}$, with values in $\mathbf{X}$, is defined by $F^{b}(t, s, u):=F(t+s, u)$ for each $u \in \mathbf{X}$.

Definition 2.4. Let $p \in[1, \infty)$. The space $B S^{p}(\mathbf{X})$ of all Stepanov bounded functions, with the exponent $p$, consists of all measurable functions $f: \mathbf{R} \mapsto \mathbf{X}$ such that $f^{b} \in L^{\infty}\left(\mathbf{R} ; L^{p}((0,1), \mathbf{X})\right)$. This is a Banach space with the norm

$$
\|f\|_{S^{p}}:=\left\|f^{b}\right\|_{L^{\infty}\left(\mathbf{R}, L^{p}\right)}=\sup _{t \in \mathbf{R}}\left(\int_{t}^{t+1}\|f(\tau)\|^{p} d \tau\right)^{1 / p} .
$$

\section{1. $S^{p}$-Pseudo Almost Periodicity}

Definition 2.5. A function $f \in C(\mathbf{R}, \mathbf{X})$ is called (Bohr) almost periodic if for each $\varepsilon>0$ there exists $l(\varepsilon)>0$ such that every interval of length $l(\varepsilon)$ contains a number $\tau$ with the property that

$$
\|f(t+\tau)-f(t)\|<\varepsilon \quad \text { for each } t \in \mathbf{R}
$$

The number $\tau$ above is called an $\varepsilon$-translation number of $f$, and the collection of all such functions will be denoted $A P(\mathbf{X})$.

Definition 2.6. A function $F \in C(\mathbf{R} \times \mathbf{Y}, \mathbf{X})$ is called (Bohr) almost periodic in $t \in \mathbf{R}$ uniformly in $y \in K$ where $K \subset \mathrm{Y}$ is any compact subset $K \subset \mathrm{Y}$ if for each $\varepsilon>0$ there exists $l(\varepsilon)$ such that every interval of length $l(\varepsilon)$ contains a number $\tau$ with the property that

$$
\|F(t+\tau, y)-F(t, y)\|<\varepsilon \quad \text { for each } t \in \mathbf{R}, y \in K \text {. }
$$

The collection of those functions is denoted by $A P(\mathbf{R} \times \mathbf{Y})$.

Define the classes of functions $P A P_{0}(\mathbf{X})$ and $P A P_{0}(\mathbf{R} \times \mathbf{X})$, respectively, as follows:

$$
P A P_{0}(\mathbf{X}):=\left\{u \in B C(\mathbf{R}, \mathbf{X}): \lim _{T \rightarrow \infty} \frac{1}{2 T} \int_{-T}^{T}\|u(s)\| d s=0\right\},
$$


and $P A P_{0}(\mathbf{R} \times \mathbf{Y})$ is the collection of all functions $F \in B C(\mathbf{R} \times \mathbf{Y}, \mathbf{X})$ such that

$$
\lim _{T \rightarrow \infty} \frac{1}{2 T} \int_{-T}^{T}\|F(t, u)\| d t=0
$$

uniformly in $u \in \mathbf{Y}$.

Definition 2.7 (see [13]). A function $f \in B C(\mathbf{R}, \mathbf{X})$ is called pseudo almost periodic if it can be expressed as $f=h+\varphi$, where $h \in A P(\mathbf{X})$ and $\varphi \in P A P_{0}(\mathbf{X})$. The collection of such functions will be denoted by $P A P(\mathbf{X})$.

Definition 2.8 (see [13]). A function $F \in C(\mathbf{R} \times \mathbf{Y}, \mathbf{X})$ is said to be pseudo almost periodic if it can be expressed as $F=G+\Phi$, where $G \in A P(\mathbf{R} \times \mathbf{Y})$ and $\phi \in P A P_{0}(\mathbf{R} \times \mathbf{Y})$. The collection of such functions will be denoted by $P A P(\mathbf{R} \times \mathbf{Y})$.

Define $A A_{0}(\mathbf{R} \times \mathbf{Y})$ as the collection of all functions $F \in B C(\mathbf{R} \times \mathbf{Y}, \mathbf{X})$ such that

$$
\lim _{T \rightarrow \infty} \frac{1}{2 T} \int_{-T}^{T}\|F(t, u)\| d t=0
$$

uniformly in $u \in K$, where $K \subset \mathrm{Y}$ is any bounded subset.

Obviously,

$$
P A P_{0}(\mathbf{R} \times \mathbf{Y}) \subset A A_{0}(\mathbf{R} \times \mathbf{Y})
$$

A weaker version of Definition 2.8 is the following.

Definition 2.9. A function $F \in C(\mathbf{R} \times \mathbf{Y}, \mathbf{X})$ is said to be B-pseudo almost periodic if it can be expressed as $F=G+\Phi$, where $G \in A P(\mathbf{R} \times \mathbf{Y})$ and $\phi \in A A_{0}(\mathbf{R} \times \mathbf{Y})$. The collection of such functions will be denoted by $B P A P(\mathbf{R} \times \mathbf{Y})$.

Definition 2.10 (see $[20,21]$ ). A function $f \in B S^{p}(\mathbf{X})$ is called $S^{p}$-pseudo almost periodic (or Stepanov-like pseudo almost periodic) if it can be expressed as $f=h+\varphi$, where $h^{b} \in A P\left(L^{p}((0,1), \mathbf{X})\right)$ and $\varphi^{b} \in P A P_{0}\left(L^{p}((0,1), \mathbf{X})\right)$. The collection of such functions will be denoted by $P A P^{p}(\mathbf{X})$.

In other words, a function $f \in L_{\mathrm{loc}}^{p}(\mathbf{R}, \mathbf{X})$ is said to be $S^{p}$-pseudo almost periodic if its Bochner transform $f^{b}: \mathbf{R} \rightarrow L^{p}((0,1), \mathbf{X})$ is pseudo almost periodic in the sense that there exist two functions $h, \varphi: \mathbf{R} \mapsto \mathbf{X}$ such that $f=h+\varphi$, where $h^{b} \in A P\left(L^{p}((0,1), \mathbf{X})\right)$ and $\varphi^{b} \in P A P_{0}\left(L^{p}((0,1), \mathbf{X})\right)$.

To define the notion of $S^{p}$-pseudo almost automorphy for functions of the form $F$ : $\mathbf{R} \times \mathbf{Y} \mapsto \mathbf{Y}$, we need to define the $S^{p}$-pseudo almost periodicity for these functions as follows.

Definition 2.11. A function $F: \mathbf{R} \times \mathbf{Y} \mapsto \mathbf{X},(t, u) \mapsto F(t, u)$ with $F(\cdot, u) \in L_{\mathrm{loc}}^{p}(\mathbf{R}, \mathbf{X})$ for each $u \in \mathbf{X}$, is said to be $S^{p}$-pseudo almost periodic if there exist two functions $H, \Phi: \mathbf{R} \times \mathbf{Y} \mapsto \mathbf{X}$ such that $F=H+\Phi$, where $H^{b} \in A P\left(\mathbf{R} \times L^{p}((0,1), \mathbf{X})\right)$ and $\Phi^{b} \in A A_{0}\left(\mathbf{R} \times L^{p}((0,1), \mathbf{X})\right)$. 
The collection of those $S^{p}$-pseudo almost periodic functions $F: \mathbf{R} \times \mathbf{Y} \mapsto \mathbf{X}$ will be denoted $P A P^{p}(\mathbf{R} \times \mathbf{Y})$.

\section{2. $S^{p}$-Almost Automorphy}

The notion of $S^{p}$-almost automorphy is a new notion due to N'Guérékata and Pankov [22].

Definition 2.12 (Bochner). A function $f \in C(\mathbf{R}, \mathbf{X})$ is said to be almost automorphic if for every sequence of real numbers $\left(s_{n}^{\prime}\right)_{n \in \mathbb{N}}$ there exists a subsequence $\left(s_{n}\right)_{n \in \mathbb{N}}$ such that

$$
g(t):=\lim _{n \rightarrow \infty} f\left(t+s_{n}\right)
$$

is well defined for each $t \in \mathbf{R}$, and

$$
\lim _{n \rightarrow \infty} g\left(t-s_{n}\right)=f(t)
$$

for each $t \in \mathbf{R}$.

Remark 2.13. The function $g$ in Definition 2.12 is measurable but not necessarily continuous. Moreover, if $g$ is continuous, then $f$ is uniformly continuous. If the convergence above is uniform in $t \in \mathbf{R}$, then $f$ is almost periodic. Denote by $A A(\mathbf{X})$ the collection of all almost automorphic functions $\mathbf{R} \rightarrow \mathbf{X}$. Note that $A A(\mathbf{X})$ equipped with the sup norm, $\|\cdot\|_{\infty}$, turns out to be a Banach space.

We will denote by $A A_{u}(\mathbf{X})$ the closed subspace of all functions $f \in A A(\mathbf{X})$ with $g \in C(\mathbf{R}, \mathbf{X})$. Equivalently, $f \in A A_{u}(\mathbf{X})$ if and only if $f$ is almost automorphic, and the convergences in Definition 2.12 are uniform on compact intervals, that is, in the Fréchet space $C(\mathbf{R}, \mathbf{X})$. Indeed, if $f$ is almost automorphic, then its range is relatively compact. Obviously, the following inclusions hold:

$$
A P(\mathbf{X}) \subset A A_{u}(\mathbf{X}) \subset A A(\mathbf{X}) \subset B C(\mathbf{X})
$$

Definition 2.14 (see [22]). The space $A S^{p}(\mathbf{X})$ of Stepanov-like almost automorphic functions (or $S^{p}$-almost automorphic) consists of all $f \in B S^{p}(\mathbf{X})$ such that $f^{b} \in A A\left(L^{p}(0,1 ; \mathbf{X})\right.$ ). That is, a function $f \in L_{\text {loc }}^{p}(\mathbf{R} ; \mathbf{X})$ is said to be $S^{p}$-almost automorphic if its Bochner transform $f^{b}$ : $\mathbf{R} \rightarrow L^{p}(0,1 ; \mathbf{X})$ is almost automorphic in the sense that for every sequence of real numbers $\left(s_{n}^{\prime}\right)_{n \in \mathbb{N}}$ there exists a subsequence $\left(s_{n}\right)_{n \in \mathbb{N}}$ and a function $g \in L_{\mathrm{loc}}^{p}(\mathbf{R} ; \mathbf{X})$ such that

$$
\begin{aligned}
& {\left[\int_{t}^{t+1}\left\|f\left(s_{n}+s\right)-g(s)\right\|^{p} d s\right]^{1 / p} \longrightarrow 0} \\
& {\left[\int_{t}^{t+1}\left\|g\left(s-s_{n}\right)-f(s)\right\|^{p} d s\right]^{1 / p} \longrightarrow 0}
\end{aligned}
$$

as $n \rightarrow \infty$ pointwise on $\mathbf{R}$. 
Remark 2.15. It is clear that if $1 \leq p<q<\infty$ and $f \in L_{\text {loc }}^{q}(\mathbf{R} ; \mathbf{X})$ is $S^{q}$-almost automorphic, then $f$ is $S^{p}$-almost automorphic. Also if $f \in A A(\mathbf{X})$, then $f$ is $S^{p}$-almost automorphic for any $1 \leq p<\infty$. Moreover, it is clear that $f \in A A_{u}(\mathbf{X})$ if and only if $f^{b} \in A A\left(L^{\infty}(0,1 ; \mathbf{X})\right)$. Thus, $A A_{u}(\mathbf{X})$ can be considered as $A S^{\infty}(\mathbf{X})$.

Definition 2.16. A function $F: \mathbf{R} \times \mathbf{Y} \mapsto \mathbf{X},(t, u) \mapsto F(t, u)$ with $F(\cdot, u) \in L_{\mathrm{loc}}^{p}(\mathbf{R} ; \mathbf{X})$ for each $u \in \mathbf{Y}$, is said to be $S^{p}$-almost automorphic in $t \in \mathbf{R}$ uniformly in $u \in \mathbf{Y}$ if $t \mapsto F(t, u)$ is $S^{p_{-}}$ almost automorphic for each $u \in \mathbf{Y}$; that is, for every sequence of real numbers $\left(s_{n}^{\prime}\right)_{n \in \mathbb{N}}$, there exists a subsequence $\left(s_{n}\right)_{n \in \mathbb{N}}$ and a function $G(\cdot, u) \in L_{\text {loc }}^{p}(\mathbf{R} ; \mathbf{X})$ such that

$$
\begin{aligned}
& {\left[\int_{t}^{t+1}\left\|F\left(s_{n}+s, u\right)-G(s, u)\right\|^{p} d s\right]^{1 / p} \longrightarrow 0} \\
& {\left[\int_{t}^{t+1}\left\|G\left(s-s_{n}, u\right)-F(s, u)\right\|^{p} d s\right]^{1 / p} \longrightarrow 0}
\end{aligned}
$$

as $n \rightarrow \infty$ pointwise on $\mathbf{R}$ for each $u \in \mathbf{Y}$.

The collection of those $S^{p}$-almost automorphic functions $F: \mathbf{R} \times \mathbf{Y} \mapsto \mathbf{X}$ will be denoted by $A S^{p}(\mathbf{R} \times \mathbf{Y})$.

\subsection{Pseudo Almost Automorphy}

The notion of pseudo almost automorphy is a new notion due to Liang et al. [2,9].

Definition 2.17. A function $f \in C(\mathbf{R}, \mathbf{X})$ is called pseudo almost automorphic if it can be expressed as $f=h+\varphi$, where $h \in A A(\mathbf{X})$ and $\varphi \in P A P_{0}(\mathbf{X})$. The collection of such functions will be denoted by $P A A(\mathbf{X})$.

Obviously, the following inclusions hold:

$$
A P(\mathbf{X}) \subset P A P(\mathbf{X}) \subset P A A(\mathbf{X}), \quad A P(\mathbf{X}) \subset A A(\mathbf{X}) \subset P A A(\mathbf{X}) .
$$

Definition 2.18. A function $F \in C(\mathbf{R} \times \mathbf{Y}, \mathbf{X})$ is said to be pseudo almost automorphic if it can be expressed as $F=G+\Phi$, where $G \in A A(\mathbf{R} \times \mathbf{Y})$ and $\varphi \in A A_{0}(\mathbf{R} \times \mathbf{Y})$. The collection of such functions will be denoted by $P A A(\mathbf{R} \times \mathbf{Y})$.

A substantial result is the next theorem, which is due to Liang et al. [2].

Theorem 2.19 (see [2]). The space PAA(X) equipped with the sup norm $\|\cdot\|_{\infty}$ is a Banach space.

We also have the following composition result.

Theorem 2.20 (see [2]). If $f: \mathbf{R} \times \mathbf{Y} \mapsto \mathbf{X}$ belongs to $P A A(\mathbf{R} \times \mathbf{Y})$ and if $x \mapsto f(t, x)$ is uniformly continuous on any bounded subset $K$ of $\mathbf{Y}$ for each $t \in \mathbf{R}$, then the function defined by $h(t)=f(t, \varphi(t))$ belongs to $P A A(\mathbf{X})$ provided $\varphi \in P A A(\mathbf{Y})$. 


\section{3. $S^{p}$-Pseudo Almost Automorphy}

This section is devoted to the notion of $S^{p}$-pseudo almost automorphy. Such a concept is completely new and is due to Diagana [7].

Definition 3.1 (see [7]). A function $f \in B S^{p}(\mathbf{X})$ is called $S^{p}$-pseudo almost automorphic (or Stepanov-like pseudo almost automorphic) if it can be expressed as

$$
f=h+\varphi,
$$

where $h^{b} \in A A\left(L^{p}((0,1), \mathbf{X})\right)$ and $\varphi^{b} \in P A P_{0}\left(L^{p}((0,1), \mathbf{X})\right)$. The collection of such functions will be denoted by $P A A^{p}(\mathbf{X})$.

Clearly, a function $f \in L_{\mathrm{loc}}^{p}(\mathbf{R}, \mathbf{X})$ is said to be $S^{p}$-pseudo almost automorphic if its Bochner transform $f^{b}: \mathbf{R} \rightarrow L^{p}((0,1), \mathbf{X})$ is pseudo almost automorphic in the sense that there exist two functions $h, \varphi: \mathbf{R} \mapsto \mathbf{X}$ such that $f=h+\varphi$, where $h^{b} \in A A\left(L^{p}((0,1), \mathbf{X})\right)$ and $\varphi^{b} \in P A P_{0}\left(L^{p}((0,1), \mathbf{X})\right)$.

Theorem 3.2 (see [7]). If $f \in P A A(\mathbf{X})$, then $f \in P A A^{p}(\mathbf{X})$ for each $1 \leq p<\infty$. In other words, $P A A(\mathbf{X}) \subset P A A^{p}(\mathbf{X})$.

Obviously, the following inclusions hold:

$$
\begin{gathered}
A P(\mathbf{X}) \subset P A P(\mathbf{X}) \subset P A A(\mathbf{X}) \subset P A A^{p}(\mathbf{X}), \\
A P(\mathbf{X}) \subset A A(\mathbf{X}) \subset P A A(\mathbf{X}) \subset P A A^{p}(\mathbf{X}) .
\end{gathered}
$$

Theorem 3.3 (see [7]). The space $P A A^{p}(\mathbf{X})$ equipped with the norm $\|\cdot\|_{S^{p}}$ is a Banach space.

Definition 3.4. A function $F: \mathbf{R} \times \mathbf{Y} \mapsto \mathbf{X},(t, u) \mapsto F(t, u)$ with $F(\cdot, u) \in L^{p}(\mathbf{R}, \mathbf{X})$ for each $u \in \mathbf{Y}$, is said to be $S^{p}$-pseudo almost automorphic if there exists two functions $H, \Phi: \mathbf{R} \times \mathbf{Y} \mapsto \mathbf{X}$ such that

$$
F=H+\Phi
$$

where $H^{b} \in A A\left(\mathbf{R} \times L^{p}((0,1), \mathbf{X})\right)$ and $\Phi^{b} \in A A_{0}\left(\mathbf{R} \times L^{p}((0,1), \mathbf{X})\right)$. The collection of those $S^{p}$-pseudo almost automorphic functions will be denoted by $P A A^{p}(\mathbf{R} \times \mathbf{Y})$.

We have the following composition theorems.

Theorem 3.5. Let $F: \mathbf{R} \times \mathbf{X} \mapsto \mathbf{X}$ be a $S^{p}$-pseudo almost automorphic function. Suppose that $F(t, u)$ is Lipschitzian in $u \in \mathbf{X}$ uniformly in $t \in \mathbf{R}$; that is there exists $L>0$ such

$$
\|F(t, u)-F(t, v)\| \leq L \cdot\|u-v\|
$$

for all $t \in \mathbf{R},(u, v) \in \mathbf{X} \times \mathbf{X}$.

If $\phi \in P A A^{p}(\mathbf{X})$, then $\Gamma: \mathbf{R} \rightarrow \mathbf{X}$ defined by $\Gamma(\cdot):=F(\cdot, \phi(\cdot))$ belongs to $P A A^{p}(\mathbf{X})$. 
Proof. Let $F=H+\Phi$, where $H^{b} \in A A\left(\mathbf{R} \times L^{p}((0,1), \mathbf{X})\right)$ and $\Phi^{b} \in A A_{0}\left(\mathbf{R} \times L^{p}((0,1), \mathbf{X})\right)$. Similarly, let $\phi=\phi_{1}+\phi_{2}$, where $\phi_{1}^{b} \in A A\left(L^{p}((0,1), \mathbf{X})\right)$ and $\phi_{2}^{b} \in P A P_{0}\left(L^{p}((0,1), \mathbf{X})\right)$, that is,

$$
\lim _{T \rightarrow \infty} \frac{1}{2 T} \int_{-T}^{T}\left(\int_{t}^{t+1}\left\|\varphi_{2}(\sigma)\right\|^{p} d \sigma\right)^{1 / p} d t=0
$$

for all $t \in \mathbf{R}$.

It is obvious to see that $F^{b}(\cdot, \phi(\cdot)): \mathbf{R} \mapsto L^{p}((0,1), \mathbf{X})$. Now decompose $F^{b}$ as follows:

$$
\begin{aligned}
F^{b}(\cdot, \phi(\cdot)) & =H^{b}\left(\cdot, \phi_{1}(\cdot)\right)+F^{b}(\cdot, \phi(\cdot))-H^{b}\left(\cdot, \phi_{1}(\cdot)\right) \\
& =H^{b}\left(\cdot, \phi_{1}(\cdot)\right)+F^{b}(\cdot, \phi(\cdot))-F^{b}\left(\cdot, \phi_{1}(\cdot)\right)+\Phi^{b}\left(\cdot, \phi_{1}(\cdot)\right) .
\end{aligned}
$$

Using the theorem of composition of almost automorphic functions, it is easy to see that $H^{b}\left(\cdot, \phi_{1}(\cdot)\right) \in A A\left(L^{p}((0,1), \mathbf{X})\right)$. Now, set

$$
G^{b}(\cdot):=F^{b}(\cdot, \phi(\cdot))-F^{b}\left(\cdot, \phi_{1}(\cdot)\right)
$$

Clearly, $G^{b}(\cdot) \in P A P_{0}\left(L^{p}((0,1), \mathbf{X})\right)$. Indeed, we have

$$
\begin{aligned}
\int_{t}^{t+1}\|G(\sigma)\|^{p} d \sigma & =\int_{t}^{t+1}\left\|F(\sigma, \phi(\sigma))-F\left(\sigma, \phi_{1}(\sigma)\right)\right\|^{p} d \sigma \\
& \leq L^{p} \int_{t}^{t+1}\left\|\phi(\sigma)-\phi_{1}(\sigma)\right\|^{p} d \sigma \\
& =L^{p} \int_{t}^{t+1}\left\|\phi_{2}(\sigma)\right\|^{p} d \sigma,
\end{aligned}
$$

and hence for $T>0$,

$$
\frac{1}{2 T} \int_{-T}^{T}\left(\int_{t}^{t+1}\|G(\sigma)\|^{p} d \sigma\right)^{1 / p} d t \leq \frac{L}{2 T} \int_{-T}^{T}\left(\int_{t}^{t+1}\left\|\phi_{2}(\sigma)\right\|^{p} d \sigma\right)^{1 / p} d t .
$$

Now using (3.5), it follows that

$$
\lim _{T \rightarrow \infty} \frac{1}{2 T} \int_{-T}^{T}\left(\int_{t}^{t+1}\|G(\sigma)\|^{p} d \sigma\right)^{1 / p} d t=0
$$

Using the theorem of composition of functions of $P A P\left(L^{p}((0,1), \mathbf{X})\right)$ (see [13]) it is easy to see that $\Phi^{b}\left(\cdot, \phi_{1}(\cdot)\right) \in P A P_{0}\left(L^{p}((0,1), \mathbf{X})\right)$.

Theorem 3.6. Let $F=H+\Phi: \mathbf{R} \times \mathbf{X} \mapsto \mathbf{X}$ be an $S^{p}$-pseudo almost automorphic function, where $H^{b} \in A A\left(\mathbf{R} \times L^{p}((0,1), \mathbf{X})\right)$ and $\Phi^{b} \in A A_{0}\left(\mathbf{R} \times L^{p}((0,1), \mathbf{X})\right)$. Suppose that $F(t, u)$ and $\Phi(t, x)$ are 
uniformly continuous in every bounded subset $K \subset \mathbf{X}$ uniformly for $t \in \mathbf{R}$. If $g \in P A A^{p}(\mathbf{X})$, then $\Gamma: \mathbf{R} \rightarrow \mathbf{X}$ defined by $\Gamma(\cdot):=F(\cdot, g(\cdot))$ belongs to $P A A^{p}(\mathbf{X})$.

Proof. Let $F=H+\Phi$, where $H^{b} \in A A\left(\mathbf{R} \times L^{p}((0,1), \mathbf{X})\right)$ and $\Phi^{b} \in A A_{0}\left(\mathbf{R} \times L^{p}((0,1), \mathbf{X})\right)$. Similarly, let $g=\phi_{1}+\phi_{2}$, where $\phi_{1}^{b} \in A A\left(L^{p}((0,1), \mathbf{X})\right)$ and $\phi_{2}^{b} \in P A P_{0}\left(L^{p}((0,1), \mathbf{X})\right)$.

It is obvious to see that $F^{b}(\cdot, g(\cdot)): \mathbf{R} \mapsto L^{p}((0,1), \mathbf{X})$. Now decompose $F^{b}$ as follows:

$$
\begin{aligned}
F^{b}(\cdot, g(\cdot)) & =H^{b}\left(\cdot, \phi_{1}(\cdot)\right)+F^{b}(\cdot, g(\cdot))-H^{b}\left(\cdot, \phi_{1}(\cdot)\right) \\
& =H^{b}\left(\cdot, \phi_{1}(\cdot)\right)+F^{b}(\cdot, g(\cdot))-F^{b}\left(\cdot, \phi_{1}(\cdot)\right)+\Phi^{b}\left(\cdot, \phi_{1}(\cdot)\right) .
\end{aligned}
$$

Using the theorem of composition of almost automorphic functions, it is easy to see that $H^{b}\left(\cdot, \phi_{1}(\cdot)\right) \in A A\left(L^{p}((0,1), \mathbf{X})\right)$. Now, set

$$
G^{b}(\cdot):=F^{b}(\cdot, g(\cdot))-F^{b}\left(\cdot, \phi_{1}(\cdot)\right)
$$

We claim that $G^{b}(\cdot) \in P A P_{0}\left(L^{p}((0,1), \mathbf{X})\right)$. First of all, note that the uniformly continuity of $F$ on bounded subsets $K \subset \mathbf{X}$ yields the uniform continuity of its Bohr transform $F^{b}$ on bounded subsets of $\mathbf{X}$. Since both $g, \phi_{1}$ are bounded functions, it follows that there exists $K \subset \mathbf{X}$ a bounded subset such that $g(\sigma), \phi_{1}(\sigma) \in K$ for each $\sigma \in \mathbf{R}$. Now from the uniform continuity of $F^{b}$ on bounded subsets of $\mathbf{X}$, it obviously follows that $F^{b}$ is uniformly continuous on $K$ uniformly for each $t \in \mathbf{R}$. Therefore for every $\varepsilon>0$ there exists $\delta>0$ such that for all $X, Y \in K$ with $\|X-Y\|<\delta$ yield

$$
\left\|F^{b}(\sigma, X)-F^{b}(\sigma, X)\right\|<\varepsilon \quad \forall \sigma \in \mathbf{R} .
$$

Using the proof of the composition theorem [2, Theorem 2.4], (applied to $F^{b}$ ) it follows

$$
\lim _{T \rightarrow \infty} \frac{1}{2 T} \int_{-T}^{T}\left(\int_{t}^{t+1}\|G(\sigma)\|^{p} d \sigma\right)^{1 / p} d t=0
$$

Using the theorem of composition [2, Theorem 2.4] for functions of $P A P_{0}\left(L^{p}((0,1), \mathbf{X})\right)$ it is easy to see that $\Phi^{b}\left(\cdot, \phi_{1}(\cdot)\right) \in P A P_{0}\left(L^{p}((0,1), \mathbf{X})\right)$.

\section{Sectorial Linear Operators}

Definition 4.1. A linear operator $A: D(A) \subset \mathbf{X} \mapsto \mathbf{X}$ (not necessarily densely defined) is said to be sectorial if the following holds: there exist constants $\omega \in \mathbf{R}, \theta \in(\pi / 2, \pi)$, and $M>0$ such that $\rho(A) \supset S_{\theta, \omega}$,

$$
\begin{gathered}
S_{\theta, \omega}:=\{\lambda \in \mathbb{C}: \lambda \neq \omega, \quad|\arg (\lambda-\omega)|<\theta\}, \\
\|R(\lambda, A)\| \leq \frac{M}{|\lambda-\omega|}, \quad \lambda \in S_{\theta, \omega} .
\end{gathered}
$$


The class of sectorial operators is very rich and contains most of classical operators encountered in literature.

Example 4.2. Let $p \geq 1$ and let $\Omega \subset \mathbf{R}^{d}$ be open bounded subset with regular boundary $\partial \Omega$. Let $\mathbf{X}:=\left(\mathbf{L}^{p}(\Omega),\|\cdot\|_{p}\right)$ be the Lebesgue space.

Define the linear operator $A$ as follows:

$$
D(A)=W^{2, p}(\Omega) \cap W_{0}^{1, p}(\Omega), \quad A(\varphi)=\Delta \varphi, \forall \varphi \in D(A) .
$$

It can be checked that the operator $A$ is sectorial on $L^{p}(\Omega)$.

It is wellknown that [14] if $A$ is sectorial, then it generates an analytic semigroup $(T(t))_{t \geq 0}$, which maps $(0, \infty)$ into $B(\mathbf{X})$ and such that there exist $M_{0}, M_{1}>0$ with

$$
\begin{gathered}
\|T(t)\| \leq M_{0} e^{\omega t}, \quad t>0, \\
\|t(A-\omega) T(t)\| \leq M_{1} e^{\omega t}, \quad t>0 .
\end{gathered}
$$

Throughout the rest of the paper, we suppose that the semigroup $(T(t))_{t \geq 0}$ is hyperbolic; that is, there exist a projection $P$ and constants $M, \delta>0$ such that $T(t)$ commutes with $P, N(P)$ is invariant with respect to $T(t), T(t): R(Q) \mapsto R(Q)$ is invertible, and the following hold:

$$
\begin{array}{cc}
\|T(t) P x\| \leq M e^{-\delta t}\|x\| & \text { for } t \geq 0, \\
\|T(t) Q x\| \leq M e^{\delta t}\|x\| & \text { for } t \leq 0,
\end{array}
$$

where $Q:=I-P$ and, for $t \leq 0, T(t):=(T(-t))^{-1}$. if

Recall that the analytic semigroup $(T(t))_{t \geq 0}$ associated with $A$ is hyperbolic if and only

$$
\sigma(A) \cap i \mathbf{R}=\emptyset,
$$

see details in [23, Proposition 1.15, page 305]

Definition 4.3. Let $\alpha \in(0,1)$. A Banach space $\left(\mathbf{X}_{\alpha},\|\cdot\|_{\alpha}\right)$ is said to be an intermediate space between $D(A)$ and $\mathbf{X}$, or a space of class $\partial_{\alpha}$, if $D(A) \subset \mathbf{X}_{\alpha} \subset \mathbf{X}$, and there is a constant $c>0$ such that

$$
\|x\|_{\alpha} \leq c\|x\|^{1-\alpha}\|x\|_{A}^{\alpha}, \quad x \in D(A),
$$

where $\|\cdot\|_{A}$ is the graph norm of $A$. 
Concrete examples of $\mathbf{X}_{\alpha}$ include $D\left(\left(-A^{\alpha}\right)\right)$ for $\alpha \in(0,1)$, the domains of the fractional powers of $A$, the real interpolation spaces $D_{A}(\alpha, \infty), \alpha \in(0,1)$, defined as the space of all $x \in \mathrm{X}$ such

$$
[x]_{\alpha}=\sup _{0<t \leq 1}\left\|t^{1-\alpha} A T(t) x\right\|<\infty
$$

with the norm

$$
\|x\|_{\alpha}=\|x\|+[x]_{\alpha}
$$

the abstract Hölder spaces $D_{A}(\alpha):=\overline{D(A)}^{\|\cdot\|_{\alpha}}$ as well as the complex interpolation spaces $[\mathbf{X}, D(A)]_{\alpha}$; see Lunardi [14] for details.

For a hyperbolic analytic semigroup $(T(t))_{t \geq 0}$, one can easily check that similar estimations as both (4.5) and (4.6) still hold with the $\alpha$-norms $\|\cdot\|_{\alpha}$. In fact, as the part of $A$ in $R(Q)$ is bounded, it follows from (4.6) that

$$
\|A T(t) Q x\| \leq C^{\prime} e^{\delta t}\|x\| \quad \text { for } t \leq 0 .
$$

Hence, from (4.8) there exists a constant $c(\alpha)>0$ such that

$$
\|T(t) Q x\|_{\alpha} \leq c(\alpha) e^{\delta t}\|x\| \quad \text { for } t \leq 0 .
$$

In addition to the above, the following holds:

$$
\|T(t) P x\|_{\alpha} \leq\|T(1)\|_{B\left(\mathbf{X}, X_{\alpha}\right)}\|T(t-1) P x\|, \quad t \geq 1,
$$

and hence from (4.5), one obtains

$$
\|T(t) P x\|_{\alpha} \leq M^{\prime} e^{-\delta t}\|x\|, \quad t \geq 1,
$$

where $M^{\prime}$ depends on $\alpha$. For $t \in(0,1]$, by (4.4) and (4.8),

$$
\|T(t) P x\|_{\alpha} \leq M^{\prime \prime} t^{-\alpha}\|x\| .
$$

Hence, there exist constants $M(\alpha)>0$ and $\gamma>0$ such that

$$
\|T(t) P x\|_{\alpha} \leq M(\alpha) t^{-\alpha} e^{-\gamma t}\|x\| \quad \text { for } t>0
$$

\section{Existence of Pseudo Almost Automorphic Solutions}

This section is devoted to the search of an almost automorphic solution to the partial hyperbolic differential equation (1.3). 
Definition 5.1. Let $\alpha \in(0,1)$. A bounded continuous function $u: \mathbf{R} \mapsto \mathbf{X}_{\alpha}$ is said to be a mild solution to (1.3) provided that the function $s \rightarrow A T(t-s) P f(s, B u(s))$ is integrable on $(-\infty, t), s \rightarrow A T(t-s) Q f(s, B u(s))$ is integrable on $(t, \infty)$ for each $t \in \mathbf{R}$, and

$$
\begin{aligned}
u(t)= & -f(t, B u(t))-\int_{-\infty}^{t} A T(t-s) P f(s, B u(s)) d s \\
& +\int_{t}^{\infty} A T(t-s) Q f(s, B u(s)) d s \\
& +\int_{-\infty}^{t} T(t-s) P g(s, C u(s)) d s \\
& -\int_{t}^{\infty} T(t-s) Q g(s, C u(s)) d s
\end{aligned}
$$

for all $t \in \mathbf{R}$.

Throughout the rest of the paper we denote by $\Gamma_{1}, \Gamma_{2}, \Gamma_{3}$, and $\Gamma_{4}$ the nonlinear integral operators defined by

$$
\begin{aligned}
& \left(\Gamma_{1} u\right)(t):=\int_{-\infty}^{t} A T(t-s) \operatorname{Pf}(s, B u(s)) d s, \\
& \left(\Gamma_{2} u\right)(t):=\int_{t}^{\infty} A T(t-s) Q f(s, B u(s)) d s \\
& \left(\Gamma_{3} u\right)(t):=\int_{-\infty}^{t} T(t-s) \operatorname{Pg}(s, C u(s)) d s \\
& \left(\Gamma_{4} u\right)(t):=\int_{t}^{\infty} T(t-s) Q g(s, C u(s)) d s .
\end{aligned}
$$

Let $p>1$ and let $q \geq 1$ such that $1 / p+1 / q=1$. Throughout the rest of the paper, we suppose that the operator $A$ is sectorial and generates a hyperbolic (analytic) semigroup $(T(t))_{t \geq 0}$ and requires the following assumptions.

(H.1) Let $0<\alpha<1$. Then $\mathbf{X}_{\alpha}=D\left(\left(-A^{\alpha}\right)\right)$, or $\mathbf{X}_{\alpha}=D_{A}(\alpha, p), 1 \leq p \leq \infty$, or $\mathbf{X}_{\alpha}=D_{A}(\alpha)$, or $\mathbf{X}_{\alpha}=[\mathbf{X}, D(A)]_{\alpha}$. Moreover, we assume that the linear operators $B, C: \mathbf{X}_{\alpha} \mapsto \mathbf{X}$ are bounded.

(H.2) Let $0<\alpha<\beta<1, f: \mathbf{R} \times \mathbf{X} \mapsto \mathbf{X}_{\beta}$ be an $S^{p}$-pseudo almost automorphic function in $t \in \mathbf{R}$ uniformly in $u \in \mathbf{X}$, and let $g: \mathbf{R} \times \mathbf{X} \mapsto \mathbf{X}$ be $S^{p}$-pseudo almost automorphic in $t \in \mathbf{R}$ uniformly in $u \in \mathbf{X}$. Moreover, the functions $f$, $g$ are uniformly Lipschitz with respect to the second argument in the following sense: there exists $K>0$ such that

$$
\begin{gathered}
\|f(t, u)-f(t, v)\|_{\beta} \leq K\|u-v\|, \\
\|g(t, u)-g(t, v)\| \leq K\|u-v\|
\end{gathered}
$$

for all $u, v \in \mathbf{X}$ and $t \in \mathbf{R}$. 
In order to show that $\Gamma_{1}$ and $\Gamma_{2}$ are well defined, we need the next lemma whose proof can be found in Diagana [12].

Lemma 5.2 (see [12]). Let $0<\alpha, \beta<1$. Then

$$
\begin{gathered}
\|A T(t) Q x\|_{\alpha} \leq c e^{\delta t}\|x\|_{\beta} \quad \text { for } t \leq 0, \\
\|A T(t) P x\|_{\alpha} \leq c t^{\beta-\alpha-1} e^{-\gamma t}\|x\|_{\beta}, \quad \text { for } t>0 .
\end{gathered}
$$

The proof for the pseudo almost automorphy of $\Gamma_{2} u$ is similar to that of $\Gamma_{1} u$ and hence will be omitted.

Lemma 5.3. Under assumptions (H.1)-(H.2), consider the function $\Gamma_{1} u$, for $u \in P A P^{p}\left(\mathbf{X}_{\alpha}\right)$, defined by

$$
\left(\Gamma_{1} u\right)(t):=\int_{-\infty}^{t} A T(t-s) \operatorname{Pf}(s, B u(s)) d s
$$

for each $t \in \mathbf{R}$. If

$$
L(q, \gamma, \alpha, \beta)=\sum_{n=1}^{\infty}\left[\int_{n-1}^{n} e^{-q \gamma s} s^{q(\beta-\alpha-1)} d s\right]^{1 / q}<\infty,
$$

then $\Gamma_{1} u \in P A A\left(\mathbf{X}_{\alpha}\right)$.

Remark 5.4. Note that the assumption $L(c, q, \gamma, \alpha, \beta)<\infty$ holds in several case. This is in particular the case when $\beta-\alpha>1 / p$.

Proof. Let $u \in P A A^{p}\left(\mathbf{X}_{\alpha}\right)$. Since $B \in B\left(\mathbf{X}_{\alpha}, \mathbf{X}\right)$, it follows that $B u \in P A A^{p}(\mathbf{X})$. Setting $h(t)=$ $f(t, B u(t))$ and using Theorem 3.5 it follows that $h \in P A A^{p}\left(\mathbf{X}_{\beta}\right)$. Moreover, using (5.5) it follows that

$$
\|A T(t-s) P h(s)\|_{\alpha} \leq c(t-s)^{\beta-\alpha-1} e^{-\gamma(t-s)}\|h(s)\|_{\beta}
$$

and hence the function $s \mapsto A T(t-s) P h(s)$ is integrable over $(-\infty, t)$ for each $t \in \mathbf{R}$.

Let $h=Y+Z$ where $\left.Y^{b} \in A A\left(L^{p}(0,1), \mathbf{X}_{\alpha}\right)\right)$ and $\left.Z^{b} \in P A P_{0}\left(L^{p}(0,1), \mathbf{X}_{\alpha}\right)\right)$. Define, for all $n=1,2, \ldots$, the sequence of integral operators

$$
\Gamma_{n}^{1}(t):=\int_{n-1}^{n} A T(s) P Y(t-s) d s, \quad \widehat{\Gamma}_{n}^{1}(t):=\int_{n-1}^{n} A T(s) P Z(t-s) d s
$$

for each $t \in \mathbf{R}$.

Now letting $r=t-s$, it follows that

$$
\Gamma_{n}^{1}(t)=\int_{t-n}^{t-n+1} A T(t-r) Y(r) d r \quad \forall t \in \mathbf{R} .
$$


Using Hölder's inequality and the estimate (5.8), it follows that

$$
\begin{aligned}
\left\|\Gamma_{n}^{1}(t)\right\|_{\alpha} & \leq \int_{t-n}^{t-n+1} c(t-r)^{\beta-\alpha-1} e^{-\gamma(t-r)}\|Y(r)\|_{\beta} d r \\
& =c\left[\int_{n-1}^{n} e^{-q \gamma s} s^{q(\beta-\alpha-1)} d s\right]^{1 / q}\|Y\|_{S^{p}} .
\end{aligned}
$$

Using the assumption $L(q, \gamma, \alpha, \beta)<\infty$, we then deduce from the well-known Weirstrass theorem that the series $\sum_{n=1}^{\infty} \Gamma_{n}^{1}(t)$ is uniformly convergent on R. Furthermore,

$$
\Gamma_{Y}(t)=\sum_{n=1}^{\infty} \Gamma_{n}^{1}(t)
$$

$\Gamma_{Y} \in C\left(\mathbf{R}, \mathbf{X}_{\alpha}\right)$, and

$$
\left\|\Gamma_{Y}(t)\right\|_{\alpha} \leq \sum_{n=1}^{\infty}\left\|\Gamma_{n}^{1}(t)\right\|_{\alpha} \leq c L(q, \gamma, \alpha, \beta)\|Y\|_{S^{p}}
$$

for each $t \in \mathbf{R}$.

We claim that $\left.\Gamma_{n}^{1} \in A A\left(\mathbf{X}_{\alpha}\right)\right)$. Indeed, let $\left(s_{m}\right)_{m \in \mathbb{N}}$ be a sequence of real numbers. Since $Y \in A S^{p}\left(\mathbf{X}_{\beta}\right)$, there exists a subsequence $\left(s_{m_{k}}\right)_{k \in \mathbb{N}}$ of $\left(s_{m}\right)_{m \in \mathbb{N}}$ and a function $\hat{Y} \in A S^{p}\left(\mathbf{X}_{\beta}\right)$ such that

$$
\left[\int_{t}^{t+1}\left\|Y\left(s_{m_{k}}+\sigma\right)-\hat{Y}(\sigma)\right\|_{\beta}^{p} d \sigma\right]^{1 / p} \longrightarrow 0 \quad \text { as } k \longrightarrow \infty
$$

Define

$$
\Delta_{n}^{1}(t)=\int_{n-1}^{n} A T(\xi) P \widehat{Y}(t-\xi) d \xi
$$

Set $H_{\alpha, \beta}^{\gamma}(\xi)=\xi^{\beta-\alpha-1} e^{-\gamma \xi}$ for $\xi>0$. Then using both Hölder's inequality and (5.5), we obtain

$$
\begin{aligned}
\left\|\Gamma_{n}^{1}\left(t+s_{m_{k}}\right)-\Gamma_{n}^{1}(t)\right\|_{\alpha} & =\left\|\int_{n-1}^{n} A T(\xi) P\left[Y\left(t+s_{m_{k}}-\xi\right)-\widehat{Y}(t-\xi)\right] d \xi\right\|_{\alpha} \\
& \leq c \int_{n-1}^{n} H_{\alpha, \beta}^{\gamma}(\xi)\left\|Y\left(t+s_{m_{k}}-\xi\right)-\widehat{Y}(t-\xi)\right\|_{\beta} d \xi \\
& \leq L_{c, q}^{\gamma, \alpha, \beta}\left[\int_{n-1}^{n}\left\|Y\left(t+s_{m_{k}}-\xi\right)-\widehat{Y}(t-\xi)\right\|_{\beta}^{p} d \xi\right]^{1 / p},
\end{aligned}
$$

where $L_{c, q}^{\gamma, \alpha, \beta}=c \cdot \sup _{n}\left[\int_{n-1}^{n}\left(H_{\alpha, \beta}^{c, \gamma}(s)\right)^{q} d s\right]^{1 / q}<\infty$, as $L(q, \gamma, \alpha, \beta)<\infty$. 
Obviously,

$$
\left\|\Gamma_{n}^{1}\left(t+s_{m_{k}}\right)-\Delta_{n}^{1}(t)\right\|_{\alpha} \longrightarrow 0 \quad \text { as } k \longrightarrow \infty .
$$

Similarly, we can prove that

$$
\left\|\Delta_{n}^{1}\left(t+s_{m_{k}}\right)-\Gamma_{n}^{1}(t)\right\|_{\alpha} \longrightarrow 0 \quad \text { as } k \longrightarrow \infty
$$

Therefore the sequence $\Gamma_{n}^{1} \in A A\left(\mathbf{X}_{\alpha}\right)$ for each $n$, and hence its uniform limit $\Gamma_{Y} \in A A\left(\mathbf{X}_{\alpha}\right)$.

Let us show that each $\widehat{\Gamma}_{n}^{1} \in P A P_{0}\left(\mathbf{X}_{\alpha}\right)$. Indeed,

$$
\begin{aligned}
\left\|\widehat{\Gamma}_{n}^{1}(t)\right\|_{\alpha} & \leq \int_{t-n}^{t-n+1} c(t-r)^{\beta-\alpha-1} e^{-\gamma(t-r)}\|Z(r)\|_{\beta} d r \\
& \leq c\left[\int_{n-1}^{n} e^{-q \gamma s} s^{q(\beta-\alpha-1)} d s\right]^{1 / q} \cdot\left[\int_{t-n}^{t-n+1}\|Z(s)\|_{\beta}^{p} d s\right]^{1 / p},
\end{aligned}
$$

and hence $\widehat{\Gamma}_{n}^{1} \in P A P_{0}\left(\mathbf{X}_{\alpha}\right)$, as $Z^{b} \in P A P_{0}\left(L^{p}\left((0,1), \mathbf{X}_{\alpha}\right)\right)$. Furthermore, using the assumption $L(q, \gamma, \alpha, \beta)<\infty$, we then deduce from the well-known Weirstrass theorem that the series

$$
\sum_{n=1}^{\infty} \widehat{\Gamma}_{n}^{1}(t)
$$

is uniformly convergent on $\mathbf{R}$. Moreover,

$$
\widehat{\Gamma}_{Z}(t)=\sum_{n=1}^{\infty} \widehat{\Gamma}_{n}^{1}(t)
$$

$\widehat{\Gamma}_{1} u \in C\left(\mathbf{R}, \mathbf{X}_{\alpha}\right)$, and

$$
\left\|\widehat{\Gamma}_{Z}(t)\right\|_{\alpha} \leq \sum_{n=1}^{\infty}\left\|\widehat{\Gamma}_{n}^{1}(t)\right\|_{\alpha} \leq L(c, q, \gamma, \alpha, \beta)\|Z\|_{S^{p}}
$$

for each $t \in \mathbf{R}$.

Consequently the uniform limit $\widehat{\Gamma}_{Z}(t)=\sum_{n=1}^{\infty} \widehat{\Gamma}_{n}^{1}(t) \in P A P_{0}\left(\mathbf{X}_{\alpha}\right)$; see [21, Lemma 2.5] . Therefore, $\Gamma_{1} u=\Gamma_{Y}+\widehat{\Gamma}_{Z}: \mathbf{R} \mapsto \mathbf{X}_{\alpha}$ is pseudo almost automorphic. omitted.

The proof for the almost automorphy of $\Gamma_{4} u$ is similar to that of $\Gamma_{3} u$ and hence will be 
Lemma 5.5. Under assumptions (H.1)-(H.2), consider the function $\Gamma_{3} u$, for $u \in P A A^{p}\left(\mathbf{X}_{\alpha}\right)$, defined by

$$
\left(\Gamma_{3} u\right)(t):=\int_{-\infty}^{t} T(t-s) \operatorname{Pg}(s, C u(s)) d s
$$

for each $t \in \mathbf{R}$.

$$
\text { If } M(q, \gamma, \alpha)=\sum_{n=1}^{\infty}\left[\int_{n-1}^{n} s^{-q \alpha} e^{-q \gamma s} d s\right]^{1 / q}<\infty \text {, then } \Gamma_{3} u \in P A A\left(\mathbf{X}_{\alpha}\right) .
$$

Proof. The proof is similar to that of Lemma 5.3 and hence omitted, though here we make use of the approximation (5.4) rather than (5.5).

Throughout the rest of the paper, the constant $k(\alpha)$ denotes the bound of the embedding $\mathbf{X}_{\beta} \hookrightarrow \mathbf{X}_{\alpha}$, that is,

$$
\|u\|_{\alpha} \leq k(\alpha)\|u\|_{\beta} \quad \text { for each } u \in \mathbf{X}_{\beta}
$$

Theorem 5.6. Under the previous assumptions and if assumptions (H.1)-(H.2) hold, then the evolution equation (1.3) has a unique pseudo almost automorphic solution whenever $K$ is small enough, that is,

$$
\Theta:=K \varpi\left[k(\alpha)+\frac{c}{\delta}+c \frac{\Gamma(\beta-\alpha)}{\gamma^{\beta-\alpha}}+\frac{M(\alpha) \Gamma(1-\alpha)}{\gamma^{1-\alpha}}+\frac{c(\alpha)}{\delta}\right]<1,
$$

where $\varpi=\max \left(\|B\|_{B\left(\mathbf{X}_{\alpha}, \mathbf{X}\right)},\|C\|_{B\left(\mathbf{X}_{\alpha}, \mathbf{X}\right)}\right)$.

Proof. In $P A A^{p}\left(\mathbf{X}_{\alpha}\right)$, define the operator $\mathbb{L}: P A A^{p}\left(\mathbf{X}_{\alpha}\right) \rightarrow C\left(\mathbf{R}, \mathbf{X}_{\alpha}\right)$ by setting

$$
\begin{aligned}
\mathbb{L} u(t)= & -f(t, B u(t))-\int_{-\infty}^{t} A T(t-s) P f(s, B u(s)) d s \\
& +\int_{t}^{\infty} A T(t-s) Q f(s, B u(s)) d s \\
& +\int_{-\infty}^{t} T(t-s) \operatorname{Pg}(s, C u(s)) d s \\
& -\int_{t}^{\infty} T(t-s) Q g(s, C u(s)) d s
\end{aligned}
$$

for each $t \in \mathbf{R}$.

As we have previously seen, for every $u \in P A A^{p}\left(\mathbf{X}_{\alpha}\right), f(\cdot, B u(\cdot)) \in P A A^{p}\left(\mathbf{X}_{\beta}\right) \subset$ $P A A^{p}\left(\mathbf{X}_{\alpha}\right)$. From previous assumptions one can easily see that $\mathbb{L} u$ is well defined and continuous. Moreover, from Theorem 3.5, Lemma 5.3, and Lemma 5.5 we infer that $\mathbb{L}$ maps $P A A^{p}\left(\mathbf{X}_{\alpha}\right)$ into $P A A\left(\mathbf{X}_{\alpha}\right)$. In particular, $\mathbb{L}$ maps $P A A\left(\mathbf{X}_{\alpha}\right) \subset P A A^{p}\left(\mathbf{X}_{\alpha}\right)$ into $P A A\left(\mathbf{X}_{\alpha}\right)$. To 
complete the proof one has to show that $\mathbb{L}$ has a unique fixedpoint. Let $v, w \in P A A\left(\mathbf{X}_{\alpha}\right)$. It is routine to see that

$$
\|\mathbb{L} v-\mathbb{L} w\|_{\infty, \alpha} \leq \Theta \cdot\|v-w\|_{\infty, \alpha}
$$

Therefore, by the Banach fixed-point principle, if $\Theta<1$, then $\mathbb{L}$ has a unique fixed-point, which obviously is the only pseudo almost automorphic solution to (1.3).

\section{Example}

Let $\Omega \subset \mathbf{R}^{N}(N \geq 1)$ be an open bounded subset with $C^{2}$ boundary $\partial \Omega$, and let $\mathbf{X}=\mathbf{L}^{2}(\Omega)$ equipped with its natural topology $\|\cdot\|_{\mathbf{L}^{2}(\Omega)}$.

Define the linear operator appearing in (1.3) as follows:

$$
A u=\Delta u \quad \forall u \in D(A)=\mathbf{H}_{0}^{1}(\Omega) \cap \mathbf{H}^{2}(\Omega) .
$$

The operator $A$ defined above is sectorial and hence is the infinitesimal generator of an analytic semigroup $(T(t))_{t \geq 0}$. Moreover, the semigroup $(T(t))_{t \geq 0}$ is hyperbolic as $\sigma(A) \cap i \mathbf{R}=\emptyset$.

Throughout the rest of the paper, for each $\mu \in(0,1)$, we take $\mathbf{X}_{\mu}=D\left((-\Delta)^{\mu}\right)$ equipped with its $\mu$-norm $\|\cdot\|_{\mu}$. Moreover, we let $\alpha=1 / 2$ and suppose that $1 / 2<\beta<1$. Letting $\widehat{B} u=B u=C u$ for all $u \in \mathbf{X}_{1 / 2}=D\left((-\Delta)^{1 / 2}\right)=\mathbf{H}_{0}^{1}(\Omega)$, one easily sees that both operators are bounded from $\mathbf{H}_{0}^{1}(\Omega)$ into $\mathbf{L}^{2}(\Omega)$ with $\varpi=1$.

We require the following assumption.

(H.3) Let $1 / 2<\beta<1$, let $F: \mathbf{R} \times \mathbf{H}_{0}^{1}(\Omega) \mapsto \mathbf{X}_{\beta}$ be an $S^{p}$-pseudo almost automorphic function in $t \in \mathbf{R}$ uniformly in $u \in \mathbf{H}_{0}^{1}(\Omega)$, and let $G: \mathbf{R} \times \mathbf{H}_{0}^{1}(\Omega) \mapsto \mathbf{L}^{2}(\Omega)$ be $S^{p}$-pseudo almost automorphic in $t \in \mathbf{R}$ uniformly in $u \in \mathbf{H}_{0}^{1}(\Omega)$. Moreover, the functions $F, G$ are uniformly Lipschitz with respect to the second argument in the following sense: there exists $K^{\prime}>0$ such that

$$
\begin{gathered}
\|F(t, u)-F(t, v)\|_{\beta} \leq K^{\prime}\|u-v\|_{\mathbf{L}^{2}(\Omega)}, \\
\|G(t, u)-G(t, v)\|_{\mathbf{L}^{2}(\Omega)} \leq K^{\prime}\|u-v\|_{\mathbf{L}^{2}(\Omega)}
\end{gathered}
$$

for all $u, v \in \mathbf{L}^{2}(\Omega)$ and $t \in \mathbf{R}$.

We have the following.

Theorem 6.1. Under the previous assumptions including (H.3), then the $N$-dimensional heat equation (1.1) has a unique pseudo almost automorphic solution $\varphi \in \mathbf{H}_{0}^{1}(\Omega) \cap \mathbf{H}^{2}(\Omega)$ whenever $K^{\prime}$ is small enough. 
Classical examples of the above-mentioned functions $F, G: \mathbf{R} \times \mathbf{H}_{0}^{1}(\Omega) \mapsto \mathbf{L}^{2}(\Omega)$ are given as follows:

$$
F(t, \widehat{B} u)=\frac{K e(t)}{1+|\widehat{B} u|}, \quad G(t, \widehat{B} u)=\frac{K m(t)}{1+|\widehat{B} u|}
$$

where the functions $e, m: \mathbf{R} \mapsto \mathbf{R}$ are $S^{p}$-pseudo almost automorphic.

In this particular case, the corresponding heat equation, that is,

$$
\begin{aligned}
\frac{\partial}{\partial t}\left[\varphi+\frac{K e(t)}{1+|\widehat{B} \varphi|}\right] & =\Delta \varphi+\frac{K m(t)}{1+|\widehat{B} \varphi|}, \quad t \in \mathbf{R}, x \in \Omega, \\
\varphi(t, x) & =0, \quad t \in \mathbf{R}, x \in \partial \Omega
\end{aligned}
$$

has a unique pseudo almost automorphic solution $\varphi \in \mathbf{H}_{0}^{1}(\Omega) \cap \mathbf{H}^{2}(\Omega)$ whenever $K$ is small enough.

\section{References}

[1] J. Liang, J. Zhang, and T.-J. Xiao, "Composition of pseudo almost automorphic and asymptotically almost automorphic functions," Journal of Mathematical Analysis and Applications, vol. 340, pp. 1493$1499,2008$.

[2] T.-J. Xiao, J. Liang, and J. Zhang, "Pseudo almost automorphic solutions to semilinear differential equations in banach spaces," Semigroup Forum, vol. 76, no. 3, pp. 518-524, 2008.

[3] S. Bochner, "Continuous mappings of almost automorphic and almost periodic functions," Proceedings of the National Academy of Sciences of the United States of America, vol. 52, pp. 907-910, 1964.

[4] C. Y. Zhang, "Pseudo-almost-periodic solutions of some differential equations," Journal of Mathematical Analysis and Applications, vol. 151, pp. 62-76, 1994.

[5] C. Y. Zhang, "Pseudo almost periodic solutions of some differential equations. II," Journal of Mathematical Analysis and Applications, vol. 192, pp. 543-561, 1995.

[6] C. Y. Zhang, "Integration of vector-valued pseudo-almost periodic functions," Proceedings of the American Mathematical Society, vol. 121, pp. 167-174, 1994.

[7] T. Diagana, "Existence of pseudo almost automorphic solutions to some abstract differential equations with $S^{p}$-pseudo almost automorphic coefficients," Nonlinear Analysis: Theory, Methods $\mathcal{E}$ Applications, vol. 70, no. 11, pp. 3781-3790, 2009.

[8] K. Ezzinbi, S. Fatajou, and G. M. N'Guérékata, "Pseudo almost automorphic solutions to some neutral partial functional differential equations in banach space," Nonlinear Analysis: Theory, Methods $\mathcal{E}$ Applications, vol. 71, no. 1-2, pp. 674-684, 2009.

[9] J. Liang, G. M. N'Guérékata, T.-J. Xiao, and J. Zhang, "Some properties of pseudo-almost automorphic functions and applications to abstract differential equations," Nonlinear Analysis: Theory, Methods $\mathcal{E}$ Applications, vol. 70, no. 7, pp. 2731-2735, 2009.

[10] S. Boulite, L. Maniar, and G. M. N'Guérékata, “Almost automorphic solutions for hyperbolic semilinear evolution equations," Semigroup Forum, vol. 71, pp. 231-240, 2005.

[11] T. Diagana and G. M. N'Guérékata, "Pseudo almost periodic mild solutions to hyperbolic evolution equations in intermediate banach spaces," Applicable Analysis, vol. 85, no. 6-7, pp. 769-780, 2006.

[12] T. Diagana, "Existence of pseudo almost periodic solutions to some classes of partial hyperbolic evolution equations," Electronic Journal of Qualitative Theory of Differential Equations, vol. 2007, no. 3, pp. 1-12, 2007.

[13] T. Diagana, Pseudo Almost Periodic Functions in Banach Spaces, Nova Science, New York, NY, USA, 2007.

[14] A. Lunardi, Analytic Semigroups and Optimal Regularity in Parabolic Problems, vol. 16 of Progress in Nonlinear Differential Equations and Their Applications, Birkhäuser, Basel, Switzerland, 1995. 
[15] T. Diagana, N. Henríquez, and E. Hernàndez, "Almost automorphic mild solutions to some partial neutral functional-differential equations and applications," Nonlinear Analysis: Theory, Methods $\mathcal{E}$ Applications, vol. 69, no. 5, pp. 1485-1493, 2008.

[16] T. Diagana and G. M. N'Guérékata, "Almost automorphic solutions to some classes of partial evolution equations," Applied Mathematics Letters, vol. 20, no. 4, pp. 462-466, 2007.

[17] T. Diagana, "Existence and uniqueness of pseudo-almost periodic solutions to some classes of partial evolution equations," Nonlinear Analysis: Theory, Methods \& Applications, vol. 66, no. 2, pp. 384-395, 2007.

[18] T. Diagana, "Existence of almost automorphic solutions to some neutral functional differential equations with infinite delay," Electronic Journal of Differential Equations, vol. 2008, no. 129, pp. 1-14, 2008.

[19] A. Pankov, Bounded and Almost Periodic Solutions of Nonlinear Operator Differential Equations, vol. 55 of Mathematics and Its Applications (Soviet Series), Kluwer Academic Publishers, Dordrecht, The Netherlands, 1990.

[20] T. Diagana, "Stepanov-like pseudo almost periodic functions and their applications to differential equations," Communications in Mathematical Analysis, vol. 3, no. 1, pp. 9-18, 2007.

[21] T. Diagana, "Stepanov-like pseudo-almost periodicity and its applications to some nonautonomous differential equations," Nonlinear Analysis: Theory, Methods \& Applications, vol. 69, no. 12, pp. 42774285, 2009.

[22] G. M. N'Guérékata and A. Pankov, "Stepanov-like almost automorphic functions and monotone evolution equations," Nonlinear Analysis: Theory, Methods E Applications, vol. 68, no. 9, pp. 2658-2667, 2008.

[23] K. J. Engel and R. Nagel, One-Parameter Semigroups for Linear Evolution Equations, vol. 194 of Graduate Texts in Mathematics, Springer, New York, NY, USA, 2000. 